\title{
ОБ ОДНОМ МЕТОДЕ ДЕКОМПОЗИЦИИ ДЛЯ ЗАДАЧИ РАСПРЕДЕЛЕНИЯ РЕСУРСОВ
}

Рассматривается прямая декомпозиция (без модификации целевых функций в подзадачах) одной задачи нелинейного программирования - т. н. задачи распределения ресурсов. В [1 $]$ было обращено внимание на тот факт, что эта задача в общем случае не координируема с помощью принципа прогнозирования взаимодействий $\left[{ }^{2}\right]$. В данной работе к условию координации названного принципа добавляется условие равновесного распределения ресурсов $\left[{ }^{3,4}\right]$ и доказывается достаточность совокупности этих условий для координируемости задачи. Показана также необходимость условия равновесного распределения ресурсов для применимости принципа прогнозирования взаимодействий.

1. Рассмотрим задачу распределения ресурсов в виде*

$$
\begin{gathered}
\sum_{i=1}^{n} f_{i}\left(x_{i}\right) \rightarrow \min , \\
\sum_{i=1}^{n} g_{i}\left(x_{i}\right) \leqslant a, \\
x_{i} \in X_{i} \quad(i=1, \ldots, n),
\end{gathered}
$$

где $g_{i}=\left(g_{1 i}, \ldots, g_{m i}\right), a=\left(a_{1}, \ldots, a_{m}\right)$.

Для задачи (1) определим т. н. функции взаимодействия (см. $\left.\left[{ }^{2}\right]\right)$ в виде (ср. $[1,5])$

$$
K_{i}(x)=\sum_{\substack{j=1 \\ j \neq i}}^{n} g_{j}\left(x_{j}\right) \quad(i=1, \ldots, n) .
$$

Разложим задачу (1) на следующие подзадачи $(i=1, \ldots, n)$ (ср. $[1,3,4])$

$$
\begin{aligned}
& f_{i}\left(x_{i}\right) \rightarrow \min , \\
& g_{i}\left(x_{i}\right)+\alpha_{i} \leqslant a, \\
& x_{i} \in X_{i},
\end{aligned}
$$

где $\alpha_{i}-$ вектор координирующих параметров. Обозначим $\alpha=$ $=\left(\alpha_{1}, \ldots, \alpha_{n}\right)$.

Предположим, что условия, при которых существуют седловые точки у функций Лагранжа, соответствующих задаче (1) и подзадачам (3) (при интересующих нас значениях $\alpha_{i}$ ), выполнены. Обозначим их через

* Задача нелинейного программирования в аналогичной постановке была исследована в $\left[{ }^{1,3-5}\right]$. 
$(\bar{x} ; \bar{\lambda})=\left(\bar{x}_{1}, \ldots, \bar{x}_{n} ; \bar{\lambda}_{1}, \ldots, \bar{\lambda}_{m}\right) \quad$ и $\quad\left(x_{i}\left(\alpha_{i}\right) ; \lambda^{i}\left(\alpha_{i}\right)\right)=\left(x_{i}\left(\alpha_{i}\right) ;\right.$ $\left.\lambda_{1}{ }^{i}\left(\alpha_{i}\right), \ldots, \lambda_{m}{ }_{m}\left(\alpha_{i}\right)\right)(i=1, \ldots, n)^{* *}$ соответственно.

Будем говорить (ср. $\left.\left[{ }^{2,5}\right]\right)$, что при выбранных $K_{i}(x)$ и подзадачах (3)

1) к задаче (1) применим принцип прогнозирования взаимодействий, если из предположения выполнения равенств

$$
\alpha_{i}=K_{i}(x(\alpha)) \quad(i=1, \ldots, n)
$$

следует

$$
x(\alpha)=\bar{x},
$$

где $x(\alpha)=\left(x_{1}\left(\alpha_{1}\right), \ldots, x_{n}\left(\alpha_{n}\right)\right)$;

2) задача (1) координируема с помощью принципа прогнозирования взаимодействий, если этот принцип применим и существуют векторы $\alpha_{i}(i=1, \ldots, n)$ такие, при которых равенства (4) выполняются.

В [ $\left.{ }^{1}\right]$ показано, что к рассматриваемой задаче принцип прогнозирования взаимодействий не применим, т. е. из равенства (4) не обязательно следует равенство (5).

Будем говорить (ср. $\left.\left[{ }^{3,4}\right]\right)$, что распределение ресурсов между подзадачами равновесное, если при выбранном координирующем векторе $\alpha=\left(\alpha_{1}, \ldots, \alpha_{n}\right)$ выполняются равенства

$$
\lambda^{i}\left(\alpha_{i}\right)=\lambda^{j}\left(\alpha_{j}\right) \quad(i, j=1, \ldots, n) \text {.*** }^{* *}
$$

Докажем, что, заменяя в принципе прогнозирования взаимодействий условия координации (4) системой координации

$$
\begin{aligned}
& \alpha_{i}=K_{i}(x(\alpha)), \\
& \lambda^{i}\left(\alpha_{i}\right)=\lambda^{j}\left(\alpha_{j}\right) \quad(i, j=1, \ldots, n),
\end{aligned}
$$

получим модифицированный принцип прогнозирования взаимодействий, который применим к задаче (1), и задача (1) координируема́ с помощью этого принципа.

2. Покажем, что при наших предположениях модифицированный принцип прогнозирования взаимодействий применим к задаче (1), т. е. из существования вектора $\bar{\alpha}=\left(\bar{\alpha}_{1}, \ldots, \bar{\alpha}_{n}\right)$, гарантирующего выполнение условий

$$
\begin{gathered}
\bar{\alpha}_{i}=K_{i}(x(\bar{\alpha}))=\sum_{\substack{j=1 \\
j \neq i}}^{n} g_{j}\left(x_{j}\left(\bar{\alpha}_{j}\right)\right), \\
\lambda^{i}\left(\bar{\alpha}_{i}\right)=\lambda^{j}\left(\bar{\alpha}_{j}\right)=\hat{\lambda} \quad(i, j=1, \ldots, n),
\end{gathered}
$$

следует, что вектор $x(\bar{\alpha})$ является решением задачи $(1)$.

Условия седловой точки для подзадачи (3) при $\alpha_{i}=\bar{\alpha}_{i}$ выражаются в виде

$$
\begin{aligned}
& f_{i}\left(x_{i}\left(\bar{\alpha}_{i}\right)\right)+\left(\lambda^{i}, g_{i}\left(x_{i}\left(\bar{\alpha}_{i}\right)\right)+\bar{\alpha}_{i}-a\right) \leqslant \\
\leqslant & f_{i}\left(x_{i}\left(\bar{\alpha}_{i}\right)\right)+\left(\lambda^{i}\left(\bar{\alpha}_{i}\right), g_{i}\left(x_{i}\left(\bar{\alpha}_{i}\right)\right)+\bar{\alpha}_{i}-a\right) \leqslant
\end{aligned}
$$

** Мы не предполагаем здесь единственности седловых точек, т. е. в общем случае у нас имеются множества седловых точек $\bar{X} \times \bar{\Lambda}$ и $X_{i}\left(\alpha_{i}\right) \times \Lambda^{i}\left(\alpha_{i}\right)(i=1, \ldots, n)$. *** В общем виде это условие можно выразить следующим образом: $\Lambda^{1}\left(\alpha_{1}\right) \cap \ldots$ $\ldots \cap \Lambda^{n}\left(\alpha_{n}\right) \neq \varnothing$, где $\Lambda^{i}\left(\alpha_{i}\right)$, как и ранее, множество векторов оптимальных множителей Лагранжа в подзадаче (3). 


$$
\begin{gathered}
\leqslant f_{i}\left(x_{i}\right)+\left(\lambda^{i}\left(\bar{\alpha}_{i}\right), g_{i}\left(x_{i}\right)+\bar{\alpha}_{i}-a\right), \quad \forall \lambda^{i} \geqslant 0, \quad \forall x_{i} \in X_{i} \\
(i=1, \ldots, n) .
\end{gathered}
$$

Учитывая условия (8), из неравенств (9) получим

$$
\begin{aligned}
& f_{i}\left(x_{i}\left(\bar{\alpha}_{i}\right)\right)+\left(\lambda^{i}, \sum_{j=1}^{n} g_{j}\left(x_{j}\left(\bar{\alpha}_{j}\right)\right)-a\right) \leqslant \\
\leqslant & f_{i}\left(x_{i}\left(\bar{\alpha}_{i}\right)\right)+\left(\hat{\lambda}, \sum_{j=1}^{n} g_{j}\left(x_{j}\left(\bar{\alpha}_{j}\right)\right)-a\right) \leqslant \\
\leqslant & f_{i}\left(x_{i}\right)+\left(\hat{\lambda}, g_{i}\left(x_{i}\right)+\bar{\alpha}_{i}-a\right), \quad \forall \lambda^{i} \geqslant 0, \quad \forall x_{i} \in X_{i} \\
& (i=1, \ldots, n) .
\end{aligned}
$$

Просуммируем неравенства (10) по индексу $i$. Обозначим $\sum_{i=1}^{n} \lambda^{i}=\lambda$. В силу произвольности и неотрицательности векторов $\lambda^{i}(i=1, \ldots, n)$ вектор $\lambda$ также произволен и неотрицателен. Получим

$$
\begin{aligned}
& \sum_{i=1}^{n} f_{i}\left(x_{i}\left(\overline{\alpha_{i}}\right)\right)+\left(\lambda, \sum_{j=1}^{n} g_{j}\left(x_{j}\left(\overline{\alpha_{j}}\right)\right)-a\right) \leqslant \\
\leqslant & \sum_{i=1}^{n} f_{i}\left(x_{i}\left(\overline{\alpha_{i}}\right)\right)+n\left(\hat{\lambda}, \sum_{j=1}^{n} g_{j}\left(x_{j}\left(\overline{\alpha_{j}}\right)\right)-a\right) \leqslant \\
\leqslant & \sum_{i=1}^{n} f_{i}\left(x_{i}\right)+\left(\hat{\lambda}, \sum_{i=1}^{n}\left[g_{i}\left(x_{i}\right)+\overline{\alpha_{i}}-a\right]\right), \quad \forall \lambda \geqslant 0, \quad \forall x_{i} \in X_{i} \\
& \quad(i=1, \ldots, n) .
\end{aligned}
$$

Из условий седловой точки для подзадачи (3) (при $\alpha_{i}=\bar{\alpha}_{i}$ ) следует, что

$$
n\left(\hat{\lambda}, \sum_{j=1}^{n} g_{j}\left(x_{j}\left(\bar{\alpha}_{j}\right)\right)-a\right)=\left(\hat{\lambda}, \sum_{j=1}^{n} g_{j}\left(x_{j}\left(\bar{\alpha}_{j}\right)\right)-a\right)=0 .
$$

Учитывая это, получим

$$
\begin{aligned}
& \left(\hat{\lambda}, \sum_{i=1}^{n}\left[g_{i}\left(x_{i}\right)+\overline{\alpha_{i}}-a\right]\right)=\left(\hat{\lambda}, \sum_{i=1}^{n} g_{i}\left(x_{i}\right)+\sum_{i=1}^{n} \sum_{\substack{j=1 \\
j \neq i}}^{n} g_{j}\left(x_{j}\left(\bar{\alpha}_{j}\right)\right)-n a\right)= \\
= & \left(\hat{\lambda}, \sum_{i=1}^{n} g_{i}\left(x_{i}\right)-a\right)+\left(\hat{\lambda},(n-1)\left[\sum_{j=1}^{n} g_{j}\left(x_{j}\left(\bar{\alpha}_{j}\right)\right)-a\right]\right)=\left(\hat{\lambda}, \sum_{i=1}^{n} g_{i}\left(x_{i}\right)-a\right) .
\end{aligned}
$$

Итак, неравенства (11) можем представить в виде (заменив везде индекс $j$ на индекс $i$ )

$$
\begin{gathered}
\sum_{i=1}^{n} f_{i}\left(x_{i}\left(\bar{\alpha}_{i}\right)\right)+\left(\lambda, \sum_{i=1}^{n} g_{i}\left(x_{i}\left(\bar{\alpha}_{i}\right)\right)-a\right) \leqslant \\
\leqslant \sum_{i=1}^{n} f_{i}\left(x_{i}\left(\bar{\alpha}_{i}\right)\right)+\left(\hat{\lambda}, \sum_{i=1}^{n} g_{i}\left(x_{i}\left(\bar{\alpha}_{i}\right)\right)-a\right) \leqslant
\end{gathered}
$$

$$
\leqslant \sum_{i=1}^{n} f_{i}\left(x_{i}\right)+\left(\hat{\lambda}, \sum_{i=1}^{n} g_{i}\left(x_{i}\right)-a\right), \forall \lambda \geqslant 0, \forall x_{i} \in X_{i}(i=1, \ldots, n) .
$$

Неравенства (12) являются условиями седловой точки для задачи (1). Следовательно, вектор $x(\bar{\alpha})=\left(x_{1}\left(\overline{\alpha_{1}}\right), \ldots, x_{n}\left(\bar{\alpha}_{n}\right)\right)$ есть решение задачи (1), т. е. $x(\bar{\alpha})=\bar{x}$, а общий для всех подзадач (3) вектор оптимальных множителей Лагранжа $\hat{\lambda}$ является вектором оптимальных мно- 
жителей Лагранжа для задачи (1). Итак, модифицированный принцип применим.

3. Для координируемости следует показать, что система (7) имеет решение. Пусть известно решение задачи (1) $\bar{x}=\left(\bar{x}_{1}, \ldots, \bar{x}_{n}\right)$ и известен соответствующий вектор оптимальных множителей Лагранжа $\bar{\lambda}=\left(\bar{\lambda}_{1}, \ldots, \bar{\lambda}_{m}\right)$. В таком случае

$$
\begin{aligned}
& \left.\sum_{i=1}^{n} f_{i}\left(\bar{x}_{i}\right)+\left(\lambda, \sum_{i=1}^{n} g_{i}\left(\bar{x}_{i}\right)-a\right) \leqslant \sum_{i=1}^{n} f_{i}\left(\bar{x}_{i}\right)+\bar{\lambda}, \sum_{i=1}^{n} g_{i}\left(\bar{x}_{i}\right)-a\right) \leqslant \\
& \leqslant \sum_{i=1}^{n} f_{i}\left(x_{i}\right)+\left(\bar{\lambda}, \sum_{i=1}^{n} g_{i}\left(x_{i}\right)-a\right), \forall \lambda \geqslant 0, \forall x_{i} \in X_{i}(i=1, \ldots, n) .
\end{aligned}
$$

Полагая в неравенствах (13) $x_{i}=\bar{x}_{i}, i \neq k$, получим

$$
\begin{gathered}
f_{k}\left(\bar{x}_{k}\right)+\left(\lambda, \sum_{i=1}^{n} g_{i}\left(\bar{x}_{i}\right)-a\right) \leqslant f_{k}\left(\bar{x}_{k}\right)+\left(\bar{\lambda}, \sum_{i=1}^{n} g_{i}\left(\bar{x}_{i}\right)-a\right) \leqslant \\
\leqslant f_{k}\left(x_{k}\right)+\left(\bar{\lambda}, g_{k}\left(x_{k}\right)+\sum_{\substack{i=1 \\
i \neq k}}^{n} g_{i}\left(\bar{x}_{i}\right)-a\right), \quad \forall \lambda \geqslant 0, \forall x_{k} \in X_{k} .
\end{gathered}
$$

Выберем теперь $\hat{\alpha}=\left(\hat{\alpha}_{1}, \ldots, \hat{\alpha}_{n}\right)$ так, чтобы

$$
\hat{\alpha}_{i}=\sum_{\substack{j=1 \\ j \neq i}}^{n} g_{j}\left(\bar{x}_{j}\right) \quad(i=1, \ldots, n) .
$$

Из неравенств (14) следует

$$
\begin{aligned}
f_{k}\left(\bar{x}_{k}\right)+ & \left(\lambda, g_{k}\left(\bar{x}_{k}\right)+\hat{\alpha}_{k}-a\right) \leqslant f_{k}\left(\bar{x}_{k}\right)+\left(\bar{\lambda}, g_{k}\left(\bar{x}_{k}\right)+\hat{\alpha}_{k}-a\right) \leqslant \\
& \leqslant f_{k}\left(x_{k}\right)+\left(\bar{\lambda}, g_{k}\left(x_{k}\right)+\hat{\alpha}_{k}-a\right), \quad \forall \lambda \geqslant 0, \forall x_{k} \in X_{k} .
\end{aligned}
$$

Мы получили условия седловой точки для подзадачи (3) при $\alpha_{k}=\hat{\alpha}_{k}$, следовательно:

$$
\bar{x}_{k}=x_{k}\left(\hat{\alpha}_{k}\right), \quad \bar{\lambda}=\lambda^{k}\left(\hat{\alpha}_{k}\right) .
$$

Но так как индекс $k$ произволен, то последние равенства справедливы для $k=1, \ldots, n$.

В результате имеем

$$
\begin{gathered}
\hat{\alpha}_{k}=\sum_{\substack{j=1 \\
j \neq k}}^{n} g_{j}\left(x_{j}\left(\hat{\alpha}_{j}\right)\right), \\
\lambda^{k}\left(\hat{\alpha}_{k}\right)=\lambda^{j}\left(\hat{\alpha}_{j}\right) \\
(j, k=1, \ldots, n) .
\end{gathered}
$$

Таким образом, система (7) имеет решение и задача (1) координируема с помощью модифицированного принципа.

4. Аналогично последнему доказательству можно показать, что при данной декомпозиции равновесное распределение ресурсов является необходимым условием для применения принципа прогнозирования взаимодействий. Действительно, пусть у нас имеется вектор $\bar{\alpha}=$ $=\left(\bar{\alpha}_{1}, \ldots, \bar{\alpha}_{n}\right)$ такой, что 


$$
\bar{\alpha}_{i}=\sum_{\substack{j=1 \\ j \neq i}}^{n} g_{j}\left(x_{j}\left(\bar{\alpha}_{j}\right)\right) \quad(i=1, \ldots, n),
$$

и пусть вектор $x(\bar{\alpha})=\left(x_{1}\left(\overline{\alpha_{1}}\right), \ldots, x_{n}\left(\overline{\alpha_{n}}\right)\right)$ является решением задачи (1). В этом случае для пары $(x(\bar{\alpha}) ; \bar{\lambda})$ (где $\bar{\lambda}$ соответствующий вектор оптимальных множителей Лагранжа в задаче (1)) справедливо (12). Полагая в этих неравенствах $x_{i}=x_{i}\left(\bar{\alpha}_{i}\right), i \neq k$, находим

$$
\begin{gathered}
f_{k}\left(x_{k}\left(\bar{\alpha}_{k}\right)\right)+\left(\lambda, \sum_{i=1}^{n} g_{i}\left(x_{i}\left(\bar{\alpha}_{i}\right)\right)-a\right) \leqslant \\
\leqslant f_{k}\left(x_{k}\left(\bar{\alpha}_{k}\right)\right)+\left(\bar{\lambda}, \sum_{i=1}^{n} g_{i}\left(x_{i}\left(\bar{\alpha}_{i}\right)\right)-a\right) \leqslant \\
\left.\leqslant f_{k}\left(x_{k}\right)+\bar{\lambda}, g_{k}\left(x_{k}\right)+\sum_{\substack{i=1 \\
i \neq k}}^{n} g_{i}\left(x_{i}\left(\bar{\alpha}_{i}\right)\right)-a\right), \quad \forall \lambda \geqslant 0, \forall x_{k} \in X_{k} .
\end{gathered}
$$

Мы получили условия седловой точки для подзадачи (3) при $\alpha_{k}=\overline{\alpha_{k}}$, а поскольку индекс $k$ произволен, то вектор $\bar{\lambda}$ является вектором оптимальных множителей Лагранжа во всех подзадачах $(3)(i=1, \ldots, n)$. Следовательно, распределение ресурсов между подзадачами равновесное.

4. Пример (ср. $\left.\left[{ }^{2,5}\right]\right)$.

$$
\begin{gathered}
\frac{1}{2}\left(x_{1}^{2}+x_{2}^{2}\right)-\left(x_{1}+2 x_{2}\right) \rightarrow \min \\
2 x_{1}+3 x_{2} \leqslant 6 \\
x_{1}+4 x_{2} \leqslant 5 \\
x_{1} \geqslant 0, \quad x_{2} \geqslant 0
\end{gathered}
$$

Нетрудно видеть, что решением этой задачи является $\bar{x}=\left(\frac{13}{17}, \frac{18}{17}\right)$. Подзадачи

выражаются здесь в виде

$$
\begin{array}{rlrl}
\frac{1}{2} x_{1}^{2}-x_{1} & \rightarrow \min , & \frac{1}{2} x_{2}^{2}-2 x_{2} \rightarrow \min , \\
2 x_{1}+\alpha_{11} & \leqslant 6, & 3 x_{2}+\alpha_{12} \leqslant 6, \\
x_{1}+\alpha_{21} & \leqslant 5, & 4 x_{2}+\alpha_{22} \leqslant 5, \\
x_{1} \geqslant 0 ; & x_{2} \geqslant 0 .
\end{array}
$$

Условия координации (7) следующие:

$$
\begin{gathered}
\alpha_{11}=3 x_{2}\left(\alpha_{2}\right), \quad \alpha_{21}=4 x_{2}\left(\alpha_{2}\right), \quad \alpha_{12}=2 x_{1}\left(\alpha_{1}\right), \quad \alpha_{22}=x_{1}\left(\alpha_{1}\right), \\
\lambda_{1}^{1}\left(\alpha_{1}\right)=\lambda_{1}^{2}\left(\alpha_{2}\right), \quad \lambda_{2}^{1}\left(\alpha_{1}\right)=\lambda_{2}^{2}\left(\alpha_{2}\right) .
\end{gathered}
$$

Выберем $\hat{\alpha}_{11}=3, \hat{a}_{21}=4, \hat{\alpha}_{12}=2, \hat{\alpha}_{22}=1$. Понятно, что решениями подзадач являются $\hat{x}_{1}=1, \hat{x}_{2}=1$ и, следовательно, условия (17) удовлетворяются. Однако $\hat{\lambda}_{1}{ }^{1}=0, \hat{\lambda}_{2}^{1}=0, \hat{\lambda}_{1}^{2}=0, \hat{\lambda}_{2}^{2}=1 / 4$, т. е. при выбранном $\hat{\alpha}$ распределение ресурсов не равновесное.

Выберем теперь $\bar{\alpha}_{11}=\frac{3 \cdot 18}{17}, \bar{\alpha}_{21}=\frac{4 \cdot 18}{17}, \bar{\alpha}_{12}=\frac{2 \cdot 13}{17}, \bar{\alpha}_{22}=\frac{13}{17}$. Легко видеть, что 
решениями подзадач являются $\bar{x}_{1}=\frac{13}{17}, \bar{x}_{2}=\frac{18}{17}$ и условия (17) удовлетворяются.

К тому же, как это легко проверить, удовлетворяются и условия (18): $\bar{\lambda}_{1}^{1}=\bar{\lambda}_{1}^{2}=0$, $\bar{\lambda}_{2}^{1}=\bar{\lambda}_{2}^{2}=\frac{4}{17}$, т. е. распределение ресурсов равновесное.

\section{Л И Т Е Р А Т У Р А}

1. Dirickx, Y. M. I., Jennergren, L. P., Pet ers on, D. W., IEEE Trans. Syst., Man. and Cybern., SMC-3, No. 5, 514 (1973).

2. Месарович М., Мако Д., Т ак ах ар а И., Теория иерархических многоуровневых систем, М., 1973.

3. Багри нов ски й К. А., В сб.: Математические методы решения экономических задач, Новосибирск, 1971, с. 5 .

4. Ten K a te, A., Manag. Sci., 18, No. 12, 734 (1972).

5. У льм С., В сб.: Модели и методы анализа экономических целенаправленных систем, Новосибирск, 1977 , с. 71 .

Институт кибернетики

Академии наук Эстонской ССР
Поступила в редакцию $31 / \mathrm{V} 1977$

\section{T. MERESSOO}

\section{UHEST DEKOMPOSITSIOONIMEETODIST RESSURSSIDE JAOTAMISE OLESANDE JAOKS}

On vaadeldud ressursside jaotamise ülesande otsest dekompositsiooni, kusjuures alamülesannete sihifunktsioone ei ole modifitseeritud. On näidatud, et ülesande koordineerimiseks koostöö ennustamise printsiibi $\left[{ }^{2}\right]$ abil on tarvilik ja piisav tasakaalus ressursside jaotus $\left[{ }^{3}\right]$.

\section{T. MERESSOO}

\section{ON A DECOMPOSITION METHOD FOR THE RESOURCE ALLOCATION PROBLEM}

A direct decomposition of the resource allocation problem is considered. The cost functions of subproblems are not modified. It is shown that the problem is coordinable by the interaction prediction principle $\left[{ }^{2}\right]$ if the balanced allocation of resources $\left[{ }^{3}\right]$ is valid. 